\title{
A importância do e-recrutamento e seleção online no processo organizacional
}

\author{
The importance of the eletronic mediated recruitment in the organization
}

\section{Aryane Santos Henriques de Lima', Aline Andrade Rabelo²}

'Autora para correspondência. Faculdade Pio Décimo. Aracaju, Sergipe, Brasil. aryanehenriques@yahoo.com.br ${ }^{2}$ Faculdade Pio Décimo. Aracaju, Sergipe, Brasil.

RESUMO I Um dos processos realizados pelo setor de Recursos Humanos de uma organização é o Recrutamento e Seleção; esta, talvez seja uma das práticas mais realizadas pelas empresas e que possui diferentes formas a depender da necessidade e da cultura organizacional de cada uma delas, pois o que cada empresa busca na contratação de pessoas, são candidatos e futuros colaboradores aptos ao exercício da função. Surge, então, o objeto desta pesquisa, a utilização de ferramentas tecnológicas para este processo, a qual é denominada de e-recrutamento. . Portanto, o objetivo deste artigo é investigar a importância da utilização de ferramentas online no processo de Recrutamento e Seleção das organizações. E, para isto é preciso observar o uso de ferramentas online em processos de seleção à distância, como também averiguar o quanto a tecnologia pode auxiliar na aproximação com os candidatos e comparar as etapas de seleção presencialmente com as etapas online, observando as questões de tempo, economia, comunicação e disponibilidade dos candidatos em relação a cada uma delas. Desta forma, a metodologia utilizada foi a revisão sistemática da literatura e, foram pesquisados artigos científicos publicados no período de 2002 a 2014 encontrados nas bases de dados da SCIELO, PEPSIC E GOOGLE ACADÊMICO, além de livros das áreas de administração, recursos humanos e psicologia organizacional e do trabalho. Verificou-se, então, que a prática do e-recrutamento e a seleção online podem ser atendidas em diversas demandas que atualmente são apresentadas para a área organizacional e/ ou recursos humanos, além de contribuir para efetivar de forma positiva nos quesitos aproximação e facilidade de comunicação entre candidatos e empresas, como maior dinamicidade, otimização do tempo e baixo custo nos processos e resultados.

Palavras-chave: e-recrutamento, recrutamento e seleção, ferramentas online, tecnologia.
ABSTRACT | One of the processes performed by the Human Resources sector of an organization is the Recruitment and Selection; this, may be one of the most performed practices by the companies and that is different depending on the needs and culture of each organization, because what each company searches for when hiring people, are candidates and future collaborators able to execute the job. Arising, this way, the object of this research, the use of technological tools in this process, known as e-recruitment. Therefore, the objective of this article is to investigate the importance of using online tools in the Recruitment and Selection process inside the organizations. And, for that it's necessary to observe the use of online tools in distant selection processes, and also check how much technology can help being closer to candidates and compare different stages of selection in person and online, observing matters of time, economy, communication and candidates availability during each one. In this way, the methodology used was the systematic review of the literature, and scientific articles published in the period from 2002 to 2014 were found in the databases of SCIELO, PEPSIC and GOOGLE ACADEMMICO, as well as books from the areas of administration, human resources and psychology organizational and work environment. It has been proved that the use of e-recruitment and online selection can happen in many contexts that nowadays are presented in organizational and human resources areas, besides contributing in a positive way to companies being closer and communicating with candidates, more dynamically, optimizing time and having low cost in processes and results.

Keywords: e-recruitment, recruitment and selection, online tools, technology. 


\section{Introdução}

Um dos processos realizados pelo setor de Recursos Humanos de uma organização é o Recrutamento e Seleção de pessoal; esta, talvez seja uma das práticas mais realizadas pelas empresas e que possui diferentes formas a depender da necessidade e da cultura organizacional de cada uma delas, pois o que cada empresa busca na contratação de pessoas, são candidatos e futuros colaboradores aptos ao exercício da função.

Surge, então, uma das propostas mais recentes e que é tema deste artigo, a utilização de ferramentas tecnológicas para este processo, a qual é denominada de e-recrutamento. Para Reche (2011), o recrutamento online apresenta seu diferencial na economia. Considerando o investimento de baixo custo, além de apresentar redução na utilização de papel e de mão de obra.

Portanto, o objetivo deste artigo é investigar a importância da utilização de ferramentas online no processo de Recrutamento e Seleção para a área de Recursos Humanos. E, para isto é preciso observar $\circ$ uso de instrumentos tecnológicos em processos de seleção à distância, como também averiguar o quanto tais recursos podem auxiliar na aproximação com os candidatos e comparar as etapas de seleção realizadas presencialmente com as etapas realizadas online, observando as questões de tempo, economia, comunicação e disponibilidade dos candidatos em relação a cada uma delas.

É importante salientar que as etapas de recrutamento online não pretendem substituir todas as situações do recrutamento e seleção que são realizadas de forma presencial, mas sim, estas buscam melhor adequação dos processos à realidade atual das organizações; visto que, em muitas demandas as empresas não conseguem em seu mercado de trabalho local o perfil adequado à sua necessidade. Como também, grandes empresas que atuam com a configuração de matriz e filial podem necessitar de maior flexibilidade em relação à participação dos gestores das vagas no processo de seleção.

Considerando a importância já salientada na atração de talentos para as empresas atualmente, observar e pesquisar novas estratégias de recrutamento e seleção pode tornar o cotidiano das pessoas que atuam nesta área mais ágil, dinâmico e estratégico, visto que hoje as grandes empresas não buscam quantidade de trabalho, mas sim, qualidade e inovação de serviços. Desta forma, o presente artigo de revisão literária visa investigar quais são os reais benefícios desta prática de e-recrutamento para os profissionais da área de Psicologia Organizacional e Recursos Humanos.

\section{Métodos}

O presente artigo trata-se de uma revisão sistemática da literatura realizada por meio de pesquisa bibliográfica, tendo em vista que é através de uma revisão de literatura que se busca conhecer materiais e estudos já publicados, ou seja, dados, aspectos e informações que foram publicadas por outros autores e estudiosos acerca de um tema.

Contudo para realização deste estudo foram lidos cerca de 45 artigos científicos publicados, sendo utilizados e explorados no decorrer desta revisão de literatura 20 artigos e 6 livros, além de pesquisas realizadas no site do Conselho Federal de Psicologia em busca das resoluções sobre os serviços de psicologia por meio de ferramentas tecnológicas e sobre avaliação psicológica. Os artigos obtidos para estudos foram buscados no período de janeiro a novembro de 2017 através do banco de dados da SCIELO, PEPSIC e GOOGLE ACADÊMICO.

Percebendo a dificuldade para encontrar material com foco na temática de recrutamento e seleção online, as buscas foram realizadas com margem de tempo superior a seis anos da publicação e materiais escritos em português, inglês e espanhol utilizandose de palavras-chaves como: recrutamento e seleção online, processo organizacional, ferramentas online, organizações, e-recrutamento, testes psicológicos informatizados.

\section{Perspectiva geral do recrutamento e seleção de pessoal}

Recrutamento e seleção podem ser definidos como um conjunto de processos sequenciais que culminam na escolha de um candidato para desempenhar 
uma função na organização. Estes devem ser precedidos de etapas e processos que definam e caracterizem a função a preencher (Oliveira, 2005). Os dois processos fazem ambos parte de um mesmo procedimento: a introdução de novos colaboradores na organização. A seleção assume-se, contrariamente ao recrutamento, como um processo de escolha, classificação e decisão, restringindo o contingente de candidatos (Chiavenato, 2000).

Uma visão simplista do processo de Recursos Humanos referente à seleção de pessoal pode definir o processo de recrutamento como uma fase de prospecção e atração de talentos para a empresa, como também o procedimento de escolha destes para avaliação e adequação ao cargo ofertado. No entanto, em momentos recentes do mercado de trabalho e da estrutura organizacional, esta atividade do setor de Recursos Humanos tem sido considerada como uma gestão de talentos, o que envolve um entendimento mais completo de toda a estrutura de entrada e saída de pessoas da empresa para o mercado e vice-versa (Dessler, $2014)$.

De acordo com Dessler (2014), o processo de Gestão de Talentos corresponde a um processo integrado e orientado por metas, que inclui planejamento, recrutamento, desenvolvimento, gestão, bônus e remuneração de funcionários. Ou seja, para este modelo de $\mathrm{RH}$, o processo de recrutamento é único e integrado com as etapas de desenvolvimento de pessoas, seja em relação ao treinamento ou reconhecimento por bonificação das mesmas em suas atividades cotidianas.

Isto porque, o centro da ação tornou-se não somente a presença do colaborador no ambiente de trabalho, mas sim, o cumprimento das metas da organização com vistas à sua produtividade total. Assim, o processo de recrutamento assumiu nos dias atuais uma importância estratégica, onde - mesmo busca o talento adequado à necessidade da empresa e que possa contribuir e desenvolver-se ao longo do tempo juntamente com suas metas e, consequentemente, acompanhar os resultados que a empresa apresenta.

Assim, todo o processo de seleção tem início no planejamento e previsão de cargos, onde o setor de
Recursos Humanos utiliza conhecimentos e técnicas para entender a situação atual da organização, sua tendência de mudança e, com isso, a programação de pessoal que será necessário para atender a sua demanda de trabalho. Desta forma, pode-se entender a atividade de recrutamento e seleção como uma etapa do trabalho do setor de recursos humanos, a qual auxilia no desenvolvimento de um trabalho integrado e eficaz nos diferentes setores da empresa, ao longo do tempo.

De acordo com Rodrigues (2014), o mundo corporativo atual tende a buscar mais que conhecimento técnico ou disciplina no ambiente de trabalho; segundo a autora o que antes era mais valorizado, como conhecimento técnico, agora não é suficiente para tornar-se uma empresa competitiva. Isto é, o que se tornou prioridade na busca pelos talentos para uma empresa não é somente o domínio da técnica, mas sim, a melhor forma de aplicá-la.

Hoje, a busca no mercado não se limita a formação, mas também se encontra relacionada às atitudes e comportamentos que os candidatos necessitam apresentar durante 0 processo de seleção, de forma que a empresa possa reconhecer nos mesmos a capacidade de desenvolver inovações na organização.

Desta forma, ainda de acordo com Rodrigues (2014), a área de recursos humanos deve se reestruturar e se adequar às novas tendências e passa a ocupar um lugar fundamental na definição da estratégia da empresa. Neste sentido, pode-se entender como uma tendência e necessidade do setor e das pessoas que fazem parte do ramo de gestão de pessoas o desenvolvimento de estratégias de avaliação, seleção, atração e retenção de talentos, de forma que estes se encontrem nas organizações de maneira produtiva e adaptada.

Assim, não somente a retenção mostra-se importante para o desenvolvimento organizacional, mas a própria atração dos mesmos à empresa configurase como a primeira etapa do trabalho do $\mathrm{RH}$, sendo que esta deve ser realizada com planejamento e estratégia. Deste modo, a seleção de pessoas em uma empresa pode ser um processo realizado com diferentes e as mais diversas etapas, a depender da necessidade do cargo e da organização. 
Primeiramente, a seleção pode ser definida através de um processo interno, externo ou misto; nesta etapa, o setor de Recursos Humanos verifica duas possibilidades para a contratação: o aproveitamento interno na empresa, através do processo de sucessão ou a atração externa, de pessoas que não conhecem ou fazem parte do ambiente organizacional (Reche, $2011)$.

Observa-se que hoje, não somente a avaliação técnica ou capacidade técnica mostra-se como suficiente para a escolha final de uma empresa por um candidato. Assim, mesmo com a realização de provas e entrevistas de conhecimento específico, o perfil e a atitude do candidato também se encontram em julgamento pelo $\mathrm{RH}$ e pelos gestores da área. Em situações nas quais o cargo não demanda um grande domínio técnico, pode-se ter apenas a consideração pela habilidade e perfil do candidato, ou seja, tudo depende da própria pessoa e não somente da sua formação (Rodrigues, 2014).

Diferentes técnicas de seleção podem ser utilizadas durante $\circ$ processo com $\circ$ objetivo de avaliar $\circ$ candidato de maneira cuidadosa, dentre estas, se encontra o teste de aptidão técnica, onde são abordados os conhecimentos necessários para ocupar o cargo em termos de domínio de tarefas; ou seja, qual a formação ou habilidades de experiência que o candidato necessita apresentar para executar essencialmente o trabalho que the é direcionado.

No entanto, como o setor de recursos humanos ou os gestores podem avaliar, por outro lado, a capacidade produtiva, ou comportamento ou atitudes de um candidato no momento da seleção? Para isso, outros tipos de técnicas são aplicados como: entrevistas de seleção, testes de personalidade, avaliações psicológicas, testes projetivos, entre outros. Nas situações de seleção atuais, podem ser observadas diversas formas de manutenção destas técnicas, através de uma ou mais etapas onde em cada uma é realizada a avaliação do candidato em todos os seus aspectos.

Dentre estas técnicas, segundo Reche (2011), a prática de utilização de ferramentas online para - recrutamento de pessoas está se consolidando na área de Recursos Humanos. No entanto, apesar do aumento crescente de utilização da internet e do acesso facilitado às redes e à conectividade por todas as classes sociais, esta ferramenta de trabalho da área de Recursos Humanos ainda é pouco explorada pela pesquisa científica e mais utilizada na prática de grandes empresas.

Segundo dados do IBGE (Instituto Brasileiro de Geografia e Estatística) divulgados pelo site Locaweb, em seis anos o número de brasileiros com acesso à internet aumentou em 143,8\%, o que significa que quase metade da população brasileira possui alguma forma de conectividade com a rede, seja em lan houses, através de celulares, laptops, computadores pessoais ou tablets.

Neste sentido, o acesso das empresas aos possíveis candidatos às suas vagas também pode ter aumentado mais de $100 \%$, caso a mesma utilize as ferramentas de recrutamento e seleção que são disponibilizadas online. Pode-se perceber, inicialmente, uma grande amplitude de comunicação das organizações com o mercado de trabalho, visto que a maior parte destas pessoas que acessam a rede pode estar disponível à participação ou em busca de processos de seleção.

A mesma pesquisa também demonstrou que a internet tem alcançado cada vez mais espaços antes distantes em termos físicos e geográficos. Ou seja, regiões onde $\circ$ acesso e a mobilidade são mais difíceis, podem ser melhor conectadas aos grandes centros através da internet. Assim, uma vaga disponível em São Paulo, por exemplo, pode ser do interesse de alguém no Amazonas e uma ferramenta de seleção online permite a este candidato não somente conhecer informações sobre a seleção, mas também, participar desta sem necessitar se deslocar para participar do processo.

Ainda segundo Reche (2011), os benefícios que uma organização pode adquirir utilizando a internet como meio de captação, recrutamento e seleção de pessoas, são diversos, como: acesso a uma grande base de dados sobre diferentes candidatos de diversas partes; agilidade na comunicação com estes candidatos através de e-mails, mensagens instantâneas; possibilidade de candidatura e envio de currículo online; também a possibilidade de participação online através de videoconferência, 
entrevista por ferramentas de comunicação; promoção de vagas em diferentes plataformas de comunicação e, consequentemente, aumento da amplitude de divulgação da empresa.

\section{Conceito de recrutamento e e-recrutamento}

Considerando que a temática é relativamente nova pode-se encontrar diferentes pesquisadores e diferentes definições acerca do recrutamento online ou e-recrutamento. À priori, o e-recrutamento é entendido como um método que objetiva somente a atração de talentos, isto é, divulgação das vagas pela internet. Por outro lado, há autores que conceituam esta prática como uma técnica não somente de atração de candidatos, mas também gestão de base de dados, triagem dos currículos e o início do uso de ferramentas avaliativas.

Sendo o e-recrutamento concebido como uma prática tão somente de atração de futuros colaboradores, alguns autores concordam que se trata da ação de divulgar vagas de emprego, através do uso de recursos eletrônicos, a exemplo, websites de consultorias, sites coorporativos de organizações e redes sociais (Galanaki, 2002; Narayanasamy, 2011 ; Parry, 2006).

No tocante aos autores que associam 0 e-recrutamento ao conjunto de ações que envolvem divulgação, base de dados, triagem e ferramentas avaliativas iniciais, observa-se em consonância que as ferramentas tecnológicas utilizadas para comunicação das vagas também podem ser utilizadas para o cadastro a captação de currículos para fazer triagem, manutenção de dados atualizados, características e competências procuradas por meio de avaliações que integram o processo e funciona como fonte para próximas e diferentes oportunidades (Costa, 2002; Palharini et. al., 2002; Mitter \& Orlandini, 2005).

O-recrutamento, então, apesar das divergências em seus conceitos, assume-se como um processo de atrair pessoas para uma organização através da internet e que se constitui em uma ferramenta de crescimento tecnológico e como um elo de ligação de alto potencial entre as empresas e os candidatos às vagas de emprego, proporcionando baixo custo, otimização do processo e a facilidade de comunicação entre os profissionais do $\mathrm{RH} e$ colaboradores.

Segundo Reche (2011), a metodologia do e-recrutamento está cada vez mais sendo utilizada, haja vista que se encontra na internet muitos sites de procura de emprego que divulgam ofertas das empresas, fortificando a internet como a ferramenta tecnológica de ligação entre os empregadores e empregados.

Portanto, pode-se entender que as ações voltadas ao e-recrutamento permitem atrair os candidatos, realizar a triagem dos currículos à vaga ofertada, gerir os currículos e as informações e monitorar as avaliações informatizadas dos candidatos.

\section{Vantagens e desvantagens do e-recrutamento frente ao recrutamento tradicional}

Entende-se que $\circ$ recrutamento tradicional contempla a parte inicial do processo de R\&S e que é de caráter sequencial, já que uma tarefa só inicia quando a anterior for finalizada, impedindo a possibilidade de coexistência de tarefas que possam ser executadas ao mesmo tempo. Além disso, nessa perspectiva, é comum usar ferramentas, como os testes escritos, que se mostram arcaicos, quando comparados com as tecnologias atuais.

Tendo em vista o exposto, Magalhães (2007) afirma que o recrutamento tradicional é um processo lento e dispendioso para as organizações. Apesar de usar as tecnologias para auxiliar o processo, é feito sem o nível de integração e automatização que é feito no e-recrutamento. Por isso, este processo, mostrase mais eficiente, pois permite maior quantidade de atração de candidatos e divulgação das vagas ofertadas.

Compreende-se que o e-recrutamento deve funcionar como etapa complementar dos processos e não ser o único método utilizado para recrutar, ou seja, uma metodologia não deve substituir a outra. Assim, a partir da literatura estudada, podemse elencar como uma das principais vantagens do e-recrutamento, a economia nos custos e a redução do tempo do processo. 
Conforme Magalhães (2007), o tempo despendido em tarefas rotineiras como a pesquisa de candidatos, arquivo e triagem de currículos, características do recrutamento tradicional, pode ser substituído por um sistema informatizado mais eficaz, isto para as organizações é o e-recrutamento assumindo-se como uma forma mais eficiente de divulgação das ofertas de emprego, face aos canais tradicionais, dado que permite um baixo custo por contato e o alcance que atinge com a análise e tratamento das candidaturas.

O e-recrutamento também pode oferecer constante informações aos candidatos no que se refere as oportunidades de trabalho no mercado advindas de diferentes áreas e organizações. Segundo Reche (2011), o recrutamento tradicional é frequentemente publicado em anúncios de jornais e revistas que exigem números limitados de caracteres ou informações. Neste sentido, o recrutamento online pode ser considerado uma fonte de clareza e ainda uma forma dinâmica, pois pode-se divulgar links que direcionam os candidatos a conteúdos relevantes, a exemplo, de informações sobre a entidade empregadora, ao mesmo tempo que se consulta a oferta.

Os dados coletados referentes aos candidatos são em formatos padronizados, permitindo uma análise mais rápida e eficiente pelos profissionais de recursos humanos. Além disso, esta prática de cadastros informatizados dos currículos, exclui a necessidade de currículos em papéis, colaborando com a sustentabilidade, além de permitir alcançar candidatos denominados passivos, isto é, aqueles que estão empregados, mas estão dispostos a trocar de emprego havendo condições de trabalho mais atrativas (Rodrigues, 2014).

Embora $\circ$ e-recrutamento apresente vantagens significativas ao longo do processo, ainda existem alguns contras em face às práticas do recrutamento tradicional, a começar pela dificuldade em atingir determinados grupos de candidatos, a exemplo, dos cargos em níveis mais operacionais, como: serviços gerais, auxiliar de produção, porteiro, entre outros.

Segundo Rodrigues (2014), é possível que o e-recrutamento atraia candidatos que são inválidos ou passivos, que não se enquadram no perfil ou que enviam o currículo apenas para saber o seu valor no mercado ou que ainda forneçam informações falsas.

Reina (2004) considera que é de fundamental importância para validação a viabilidade dos resultados que haja controle por parte dos profissionais de recursos humanos a conciliação entre $\circ$ recrutamento online e tradicional, pois a organização deve garantir que o processo não é completamente informatizado e despersonalizado, devendo possuir objetivos a serem estabelecidos e alcançados.

A divulgação de vagas na internet permite alcançar candidatos do mundo inteiro, o que gera um grande volume de candidaturas. Segundo Rodrigues (2014) isto pode implicar em muito tempo despendido na triagem de currículos até alcançar os candidatos com as competências desejadas e a filtragem incorreta pode ocasionar uma perda de recursos à organização.

Há autores que salientam as consequências jurídicas de um recrutamento online, a exemplo, Narayanasamy (2011) destacando em sua pesquisa, as informações e critérios divulgados em anúncios virtuais, que podem levar à possíveis dados discriminatórios.

Por fim, Magalhães (2007) comenta sobre um dos argumentos mais utilizados pelos profissionais de recursos humanos, inclusive, os psicólogos organizacionais, que se refere a falta de contato humano em todo processo de e-recrutamento. Apesar da mais-valia introduzida pelos processos tecnológicos no recrutamento, o contato interpessoal considerado fundamental na escolha de candidatos mais adequados, fica inexistente.

\section{Seleção de pessoal: recursos, técnicas e métodos informatizados}

Ao falar de seleção, Martins (2007) afirma que este processo consiste na comparação entre perfis dos candidatos e os requisitos do cargo, sendo assim, o ideal é que o perfil e o cargo se ajustem. Deste modo, é essencial que se escolha a pessoa certa para o cargo certo, objetivando, assim, manter ou aumentar a eficiência e desempenho do pessoal. Evidencia-se, então, que cada etapa da seleção 
representa um momento de tomada de decisão em que se busca aumentar o conhecimento da organização sobre as experiências, habilidades e a motivação do empregado, com isso aumentam-se as informações para que seja feita a seleção final (Baylão \& Rocha, 2014).

No processo de seleção de pessoal, o principal objetivo é escolher aquele candidato que possui melhores conhecimentos e habilidades para desempenhar as atividades de determinado cargo, bem como contribuir para que a organização atinja seu objetivo, diante das exigências do mercado de trabalho (Katsurayama et. al., 2012).

As atividades de seleção, tipicamente, seguem a um padrão determinado e consistem, prioritariamente, em provas de conhecimento, com a finalidade de medir o grau de conhecimentos e habilidades que - candidato possui sobre determinados assuntos. Essas provas medem o grau de conhecimento profissional ou técnico, como noções de informática, conhecimentos gerais e específicos, redação, inglês, etc. Elas podem ser orais, escritas, práticas e online (Guimarães \& Arieira, 2005).

Para acompanhar os avanços tecnológicos e das demandas atuais surgem ferramentas para selecionar e avaliar candidatos de maneira online, no entanto, ao mesmo tempo, tais meios estão sendo utilizados de modo inadequado, em detrimento ao desconhecimento por parte dos profissionais de $\mathrm{RH}$, acrescido também da complexidade que circundam a execução dos métodos online.

Para corroborar com o exposto, Almeida (2004) alega que as diferentes terminologias para tais recursos, técnicas e métodos geram enorme confusão, além disso, o autor afirma que ainda não existe no Brasil um guia ou manual para orientar os profissionais em relação aos moldes de uso e aos benefícios dessas tecnologias.

Desta forma, afirma-se que para melhor compreensão e avaliação do uso de ferramentas tecnológicas para seleção de pessoal é necessário analisar alguns aspectos, conforme salienta Almeida (2004) os instrumentos que serão utilizados com o objetivo de observar o desempenho dos candidatos; a forma utilizada para gerenciar as informações coletadas pelo sistema aplicado ao candidato; qual o melhor modo de uso para que se realize a captação e seleção de pessoas a nível virtual.

Eis, então, algumas ferramentas elencadas como úteis que oferecem suporte para a seleção de pessoas de maneira online. A começar pela captura de currículos - sistemas que possibilitam o envio de currículos para sites de empresas ou de consultorias de $\mathrm{RH}$; Agentes de recrutamento eletrônicos de avaliação de currículos - programas que realizam a busca de candidatos qualificados a partir de consulta dos currículos disponíveis na internet em sites coorporativos e de consultorias; avaliação de qualificações - são softwares que analisam se os candidatos possuem competências desejadas para o desempenho da função, eliminando aqueles que não contemplam as exigências da vaga; Entrevista estruturada online que possibilita aos candidatos participantes uma entrevista padronizada, que está atrelada às competências exigidas pela função ofertada; Testes de conhecimento que permitem a avaliação de candidatos em suas habilidades e conhecimentos específicos das áreas oferecidas; inventários da cultura organizacional: são questões que avaliam as preferencias, valores, crenças e expectativas dos candidatos em relação ao ambiente de trabalho, ou seja, a ferramenta permite alinhar à vaga, os candidatos que correspondem ao perfil da cultura organizacional da empresa (Almeida, 2004).

Para completar, este mesmo autor ainda fala dos testes de aptidão e personalidade, que servem para avaliar os candidatos em quesitos verbal, espacial e lógico, como também as tendências comportamentais, a exemplo, estilo de liderança e capacidade de trabalhar em equipe; Testes de integridade - utilizados para verificar comportamentos inadequados (agressividade, dependência química e patologias); Simulação permite identificar o desempenho dos candidatos a partir das respostas dos mesmos frente as situações corriqueiras do ambiente de trabalho; Investigação social - busca em outras fontes sobre as informações que não foram fornecidas pelos candidatos, ou seja, consulta às cartas de referências e antecedentes criminais. 
Face ao exposto, cabe ao profissional de recursos humanos analisar quais ferramentas serão necessárias e que melhor se adaptará a realidade da sua rotina de recrutamento e seleção de pessoal utilizada na organização ou consultoria em que está inserido, utilizando-se destes recursos e métodos de forma correta e eficaz, visando a empregabilidade de ferramentas tecnológicas que otimizam o tempo de processo, sem perder a qualidade e veracidade.

\section{A psicologia frente ao processo de e-recrutamento e seleção online}

Entendendo a dinâmica organizacional, as exigências e $\circ$ imediatismo no processo de recrutamento e seleção, busca-se utilizar diversas ferramentas que facilitem a identificação de um indivíduo que apresente as características exigidas. Sendo assim, algumas destas ferramentas são entrevistas, questionários e os testes de aptidão, habilidades e de personalidade (Katsurayama et. al., 2012). Neste sentido, pode-se afirmar que parte destas ferramentas são somente utilizadas pelos profissionais formados em Psicologia.

Desta forma, diante das diversas mudanças nas relações humanas devido a evolução da tecnologia, - Conselho Federal de Psicologia - CFP tem estabelecido regras e direcionamentos para embasar e nortear o trabalho dos psicólogos que utilizam as novas tecnologias que se renovam e se transformam a cada ano. Tais intervenções na área da tecnologia foram abordadas na Resolução CFP n. $011 / 2012$, apresentando se de forma precavida e adaptativa, tendo em vista as frequentes e dinâmicas transformações que acontecem a cada ano, no campo da informática.

Dentre os serviços psicológicos que podem ser realizados por meios tecnológicos de comunicação encontram-se os processos prévios de seleção de pessoal e a aplicação de testes devidamente regulamentados por resolução pertinente. . Pode-se, então, considerar que o CFP se mostra atento devido as diversas mudanças provocadas pela evolução da tecnologia da informação e suas relações humanas.

Assim, ao tentar se adequar às novidades e tendências tecnológicas que $\circ$ mercado oferece em diversas áreas de conhecimento, a Psicologia acaba se expondo ao risco de perder um dos seus elementos principais que é o contato pessoal, já que os empresários acreditam que a utilização de softwares pode fazer o papel do psicólogo, porém por mais precisa que seja a tecnologia desenvolvida para substituir um psicólogo em campo, algumas singularidades humanas não podem ser percebidas por máquinas ou via software (Katsurayama et. al., 2012).

A utilização do processo de recrutamento e seleção de maneira informatizada está aliada ao aperfeiçoamento da prática do profissional de psicologia, ou seja, formação, competência e atualização possibilitando melhor compreensão do contexto de avaliação psicológica e interpretação mais adequada dos resultados. Portanto, essa transformação trata-se de aprimorar a formação do psicólogo, permitindo, que o profissional seja capaz de avaliar a qualidade dos instrumentos e saiba fazer o uso adequado dos mesmos (Katsurayama et. al., 2012), pois os planos de ações para qualificação do profissional envolvem capacitação e atualização dos instrumentos e técnicas.

Cabe, então, ao psicólogo ficar atento às qualidades psicométricas dos instrumentos utilizados, no que se refere à validade preditiva, rigidez metodológica e ampla fundamentação teórica, bem como sua capacitação técnica e ética no processo avaliativo. Segundo Cruz (2002), para isso, o psicólogo necessita ter um vasto conhecimento em relação às técnicas que pretende utilizar e às teorias que embasam esses recursos, assim como uma possibilidade de crítica consciente em relação aos instrumentos de avaliação que usa, para adequar o instrumento à demanda, quando necessário, obtendo, assim, um melhor resultado.

\section{Considerações finais}

A prática do e-recrutamento e a seleção online podem ser atendidas em diversas demandas que atualmente são apresentadas para a área da Psicologia Organizacional e/ou Recursos Humanos como um todo, além de contribuir para efetivar de forma positiva os aspectos apresentados no decorrer do artigo, principalmente, a questão de 
aproximação e facilidade de comunicação entre candidatos e empresas, como maior dinamicidade e otimização do tempo nos processos e resultados.

O e-recrutamento assume-se como uma tendência inovadora, como tal, nem todos os recrutadores se sentem ainda à vontade para lidar com a internet e as suas ferramentas. Empresas que não tenham os seus processos organizacionais instituídos com base na plataforma da internet, necessitam evoluir, pois para que $\circ$ e-recrutamento seja efetivamente vantajoso para as organizações, estas devem possuir, ou capacitar-se de algumas competências e habilidades.

A automatização das operações de recrutamento e seleção poderia também encarar-se como uma ferramenta estratégica de valor incomensurável para a gestão de recursos humanos. Dispondo de mecanismos tecnológicos integrados que permitissem aos gestores do recrutamento automatizar algumas das etapas do recrutamento e seleção, libertaria estes gestores de algumas tarefas rotineiras e facilmente tratadas por software adequado (Rodrigues, 2014). Ao libertar os gestores de recursos humanos deste tipo de tarefas, eles poderiam ocupar mais tempo com as questões mais ligadas à seleção dos candidatos e com a avaliação do recrutamento e integração do recrutamento na estratégia global da organização.

Mediante o estudo realizado observa-se que atualmente as empresas e profissionais do ramo de recrutamento e seleção tendem a evidenciar maior preocupação com a técnica que irão utilizar em seus procedimentos de seleção do que, realmente, com a contribuição que a mesma pode oferecer para melhorar a qualidade do trabalho e do relacionamento empresa-candidato. Assim, pode-se concluir que algumas empresas podem mostrar-se rígidas em relação às etapas do seu recrutamento, considerando que para haver uma decisão "perfeita" e escolha do candidato "perfeito" processos determinados devam ser seguidos, mesmo que estes levem a altos custos e morosidade na seleção de pessoas.

\section{Conflitos de interesses}

Nenhum conflito financeiro, legal ou político envolvendo terceiros (governo, empresas e fundações privadas, etc.) foi declarado para nenhum aspecto do trabalho submetido (incluindo mas não limitandose a subvenções e financiamentos, conselho consultivo, desenho de estudo, preparação de manuscrito, análise estatística, etc).

\section{Referências Bibliográficas}

Almeida, W. (2004). Captação e Seleção de Talentos Repensando a teoria e a prática. São Paulo: Atlas.

Baylão, A. L. S., \& Rocha, A. P. S. (2014). A Importância do Processo de Recrutamento e Seleção de Pessoal na Organização Empresarial. XI Simpósio de Excelência em Gestão e Tecnologia. Resende, RJ, Brasil. 11. Recuperado de https://www.aedb.br/seget/arquivos/ artigos 14/20320178.pdf

Chiavenato, I. (2000). Recursos Humanos. São Paulo: Atlas.

Costa, T. R. (2002). e-RH : o impacto da tecnologia para a gestão competitiva de Recursos Humanos (Trabalho de conclusão de curso). Faculdade de Economia, Administração e Contabilidade da USP, São Paulo, SP, Brasil. Recuperado de http://docplayer.com. $\mathrm{br} / 1675205$-E-rh-o-impacto-da-tecnologia-para-agestao-competitiva-de-recursos-humanos.html

Cruz, R. M. (2002). Medidas psicológicas em psicologia do trabalho e das organizações. In Cruz, R. M.; Alchieri, J. C.; Sarda Júnior, J. J. (Org.). Avaliação e medidas psicológicas: produção de conhecimento e da intervenção profissional. São Paulo: Casa do Psicólogo.

Dessler, G. (2014). Administração de Recursos Humanos. Tradução Cecília Leão Oderich. Parte 2: Equipes de trabalho: descrição de cargos e alocação de pessoal. $3^{a}$ Edição. São Paulo: Pearson Education do Brasil.

Galanaki, E. (2002). The decision to recruit online: a descriptive study. Career Development International, 7(4), 243-251. Recuperado de http://www.emeraldinsight.com/doi/ pdfplus/10.1 108/13620430210431325. doi: $10.1108 / 13620430210431325$

Guimarães, M. F., \& Arieira, J. O. (2005). O Processo de Recrutamento e Seleção como uma Ferramenta de Gestão. Rev. Ciências Empresariais da UNIPAR, 6 (2), 203-214. Recuperado de http://revistas.unipar.br/ index.php/empresarial/article/view/309/280 
Katsurayama, M., Silva, S. R., Eufrázio, W. N., Souza, R. S. A., \& Becker, M. A. Á. (2012). Testes informatizados como auxílio na seleção em recursos humanos. Psicologia: teoria e prática, 14(2), 141-151. Recuperado de http://pepsic.bvsalud.org/pdf/ptp/v14n2/ v14n2al 2.pdf

Magalhães, T. A. C. M. G. (2007). Automatização do Processo de Recrutamento e Resposta a Oportunidades Comerciais (Dissertação de mestrado). Universidade Técnica de Lisboa. Lisboa, Portugal.

Martins, J. (2007). Recursos Humanos. Recuperado de http:// w3.valg.pt/ jmartins/gestao/Final.pdf

Mitter, G. V., \& Orlandini, J. M. (2005). Recrutamento Online/Internet. Maringá Management: Revista de Ciências Empresariais, 2(2), 19-34. Recuperado de http://www. maringamanagement.com.br/novo/index.php/ojs/ article/view/17/11

Narayanasamy, P. S. (2011). Global Trends in E-recruitment and its outcomes. Akshaya International Journal of Management Studies, 01(02), 51-54.

Oliveira, L. S. (2005). Gestão de pessoal: métodos e processos de recrutamento e seleção (Trabalho de conclusão de curso). UniCEUB - Centro Universitário de Brasília. Brasília, DF, Brasil.

Palharini, F. A., Nunes, F. S., Nascimento, P. F., \& Martins, V. A. P. (2002). Recrutamento de Recursos Humanos: fundamentos e tendências. Cadernos do ICHF, (80), 1-24.

Parry, E. (2006). Drivers of the adoption of online recruitment - an analysis using diffusion of innovation theory. Cranfield School of Management, 1-13.

Portal Locaweb (2013). Dados atualizados sobre o crescimento da internet no Brasil.

Reche, G. (2011). E-recrutamento: a internet como ferramenta de recrutamento e seleção. Recuperado de http:// www.administradores.com.br/artigos/economia-efinancas/e-recrutamento-a-internet-como-ferramentano-recrutamento-e-selecao/55112/

Reina, M. P. A. (2004). Nuevos procedimientos en el proceso empresarial de provisión de candidatos: El reclutamiento on line. Cuaderos de Ciencias Económicas Y Empresariales, (47), 89-1 10. Recuperado de http:// cuadernos.uma.es/pdfs/pdf585.pdf

Rodrigues, R. I. M. (2014). E-recrutamento como opção estratégica: realidade ou quimera nas empresas da região Minho? (Dissertação de mestrado). Universidade do Minho. Braga, Portugal. Recuperado de http://repositorium.sdum.uminho.pt/ handle/1822/34956 